\title{
Impact of the nutritional status of children with congenital heart diseases on the early post-operative outcome
}

\author{
Sameh R. Ismail ${ }^{*} \mathbb{C}$, Akhter Mehmood, Noura Rabiah, Riyadh M. Abu-sulaiman and Mohamed S. Kabbani
}

\begin{abstract}
Background: Children with congenital heart disease (CHD) frequently become malnourished due to many factors including hypermetabolic state, inadequate caloric intake, malabsorption, and fluid restriction as part of the hemodynamic intervention. The abnormal nutritional status may affect early outcome after pediatric cardiac surgery; we aim to prove the importance of nutritional assessment and patient nutritional preparation before performing pediatric cardiac surgery.

Results: We conducted a prospective observational cohort study from March 2013 till January 2015. All children from birth until 14 years of age admitted to the pediatric cardiac intensive care unit (PCICU) after cardiac surgery. Nutritional status was assessed preoperatively and prognostic nutritional index (PNI) was calculated. All post-operative outcome parameters, PCICU and hospital length of stay (LOS), ventilation time, the rate of infection, and ICU mortality were recorded. Two hundred fifty-nine children were evaluated. At admission, 179 patients (69\%) had intake less than $50 \%$ of the recommended daily allowance (RDA) of calories intake. There was a statistically significant relationship between pre-admission RDA and the infection rate ( $P$ value 0.018 ). Severely malnourished patients had significantly longer ICU length of stay (LOS) ( $P$ value $=0.049)$. Similarly, weight and height percentiles have a significant effect on ICU LOS ( $P$ value 0.009 and 0.045$)$ respectively. There was a significant relation between PNI $\geq 55$ and the ICU LOS $(P<$ $0.05)$, and ventilation time $(P=0.036)$. Delay in feeding postoperatively was associated with a higher risk of infection $(P=0.005)$, and higher mortality $(P=0.03)$.

Conclusions: Children with $\mathrm{CHD}$ were significantly malnourished preoperatively and had further weight loss postoperatively. Preoperative nutritional status and delayed postoperative enteral feeding were associated with a higher infection rate and mortality.
\end{abstract}

Keywords: Congenital heart disease, Children, Nutrition, Postoperative, Early outcome

\section{Background}

Malnutrition problems are more common in infants and children as compared to adults due to a lower percentage of muscle mass and fat and high resting energy expenditure with little reserves. Children have an increased risk of developing malnutrition particularly protein malnutrition when they have a serious illness [1].

\footnotetext{
*Correspondence: samis93@gmail.com

Department of Cardiac Sciences, National Guard Hospital Health Affairs, King Abdulaziz Medical City, P.O. Box 22490, Riyadh 11426, Saudi Arabia
}

Congenital heart disease (CHD) is a serious condition. Studies show that congenital heart diseases account for more infant deaths than all other congenital defects put together [2]. Most of patients with congenital heart diseases decompensate early. Twenty percent of these children develop heart failure in the first week of life, $18 \%$ between the first and fourth week, and $20 \%$ between 1 and 12 months [3].

Several studies have reported a link between CHD and malnutrition. It has been shown that heart injuries associated with cyanosis, congestive heart failure, and 
pulmonary hypertension lead to impairment of growth velocity, weight gain, and height development [4]. Furthermore, children with CHD are commonly malnourished for multiple reasons that may include hypermetabolic state, inadequate caloric intake, malabsorption, genetic factors, or a consequence of fluid restriction as part of hemodynamic intervention [5, 6]. Some children are submitted to special diets and often present clinical alterations, such as reduced gastric capacity [7], anoxia, circulation congestion, altered intestinal motility, and decreased absorption [3]. It is wellknown that these alterations interfere with food intake, which remains below age-specific nutritional requirements leading to malnutrition $[8,9]$.

Malnutrition is a common cause of morbidity and mortality [10] in children with congenital heart disease [11, 12]. Specifically, it has a negative impact on ventilation time [11], outcome post-operatively $[13,14]$ and length of hospital stay [15]. Adequate nutrition is essential for growth, wound healing, and proper immune function [16]. PNI is a useful tool to prognosticate the risk of malnutrition in this category of patients. PNI is a simple and objective indicator of postoperative outcomes of oncology surgery patients as well as in patient with heart failure $[13,14]$. The aim of this study is to assess the nutritional status of children with CHD and evaluate its effects on morbidity and mortality outcome after cardiac surgery, and to study the validity of prognostic nutritional index (PNI) as a useful tool to prognosticate the risk of malnutrition in this category of patients.

\section{Methods}

This is a prospective observational cohort study in which consecutive patients with diagnosis of CHD of both genders from birth until 14 years of age who were admitted from March 2014 to January 2015 to PCICU after cardiac surgery were included. The pediatric cardiac ICU has an average of 400 pediatric cardiac surgery per year with $80 \%$ of them as open-heart surgery and $20 \%$ of cases are neonatal cardiac surgery. All types of cardiac surgery and mechanical circulatory support are performed in our center except for pediatric heart transplant. All patients were intubated and mechanically ventilated postoperatively and they received sedatives and analgesics with or without neuromuscular blocker (cisatracurium). Core body temperature was monitored and hyperthermia (temperature $>37.5^{\circ} \mathrm{C}$ ) was treated with antipyretics and cooling blankets as needed.

Nutritional management was prescribed by the attending physicians, who made decisions regarding energy and protein intakes, independent of the study. Maintenance fluids were adjusted according to daily fluid balance and the patient's individual needs. We measured preoperatively serum albumin, serum transferrin, total leukocyte count at admission to check nutritional status of all patients included in the study, and we calculated prognostic nutritional index (PNI) for all patients prior to surgery. A dedicated dietitian specialist was assigned to take thorough nutritional history from the parents on admission and estimated pre-admission average calories intake and compared it with the RDA and then decided whether the patient meet or does not meet the $50 \%$ of RDA. The patient was also assessed and followed up afterwards by weight for age (WFA), weight for height (WFH), and height for age (HFA). Malnutrition was further classified according to Waterlow classification as mild ( 80 to $90 \%$ of (WFH), moderate (70 to $80 \%$ of WFH), and severe (less than $70 \%$ of WFH) [17-19]. Also, we used one of the prognostic nutritional indices, which are the Onodera's PNI [20]; it is calculated using serum albumin level $(\mathrm{g} / \mathrm{dl})$, and peripheral blood total lymphocytic count (TLC). The Onodera's PNI value was then calculated using the following formula: $10 \times$ serum albu$\min (\mathrm{g} / \mathrm{dL})+0.005 \times$ total lymphocyte count $\left(\right.$ per $\left.\mathrm{mm}^{3}\right)$. We checked different levels of PNI and its relation to the postoperative outcome. Based on Center of Diseases Control (CDC) definition of healthcare-associated infections (https://www.cdc.gov/hai/infectiontypes.html), we recorded in all patients NPO days before starting feeding, the number of days needed to achieve RDA, weight gain, or loss during ICU stay. Nutritional status was further assessed for correlation with mechanical ventilation duration, infection rate, PCICU LOS, hospital LOS, ICU mortality, and hospital mortality.

We recorded in the study all types of infection that occurred during patient stay in the PCICU including blood stream infection, central line-related infection, ventilator-associated pneumonia, and urinary tract infection.

We used Pearson's correlation coefficient test to identify significant correlations of postoperative outcome with the different nutritional parameters. Differences between 2 groups were determined using the MannWhitney's $U$ test (nonparametric test), and unpaired $t$ test (parametric test). Differences with a $P$ value $<.05$ were considered statistically significant.

\section{Results}

\section{Clinical characteristics of the study population}

A total of 259 children diagnosed with CHD who underwent cardiac surgery were evaluated. Eighty percent of the patients had an open heart surgery. Eighty-eight percent were in RACHs category $(1,2$, and 3 ), while only $12 \%$ were in category $(4,5$, and 6$)$. The mean (SD) age was 19 (28.3) months, with a median of 7 months (range 0-192). Majority of patients were terms (97.5\%). The percentage 
of patients with abnormal weight or height less than 5\% were $48 \%$ and $36 \%$ of the sample respectively. Patients with gastroesophageal reflux disease (GERD) were 9.7\% of the sample population. The occurrence of constipation within the sample was $12.7 \%$ (Table 1).

Patients were categorized according to the RDA into two categories: The first category includes 179 patients (69\%) who did not reach RDA, while the second category includes the remaining 80 patients $(31 \%)$ who reached RDA (Figs. 1 and 2). Mean of total days to reach RDA was $6.1 \pm 6$ days with a median of 4 days (range 1-30). The

Table 1 Clinical characteristics of the study population

\begin{tabular}{|c|c|c|c|}
\hline Variable & & $\begin{array}{l}\text { Total number of } \\
\text { patients, } 259\end{array}$ & $\%$ \\
\hline \multirow[t]{2}{*}{ Infection } & No infection & 183 & 58.5 \\
\hline & Infection & 76 & 41.5 \\
\hline \multirow[t]{2}{*}{ Mortality } & Alive & 248 & 95.8 \\
\hline & Dead & 11 & 4.2 \\
\hline \multirow[t]{2}{*}{ Maturity } & Term & 244 & 94.2 \\
\hline & Preterm & 15 & 5.8 \\
\hline \multirow[t]{5}{*}{ Weight (percentile) } & 5 and less & 123 & 48.2 \\
\hline & $5-10$ & 28 & 10.0 \\
\hline & $10-25$ & 59 & 23.1 \\
\hline & $25-50$ & 26 & 10.2 \\
\hline & 50 and more & 19 & 7.5 \\
\hline \multirow[t]{5}{*}{ Height (percentile) } & 5 and less & 91 & 36.3 \\
\hline & $5-10$ & 22 & 8.8 \\
\hline & $10-25$ & 76 & 29.5 \\
\hline & $25-50$ & 26 & 10 \\
\hline & 50 and more & 40 & 15.5 \\
\hline \multirow[t]{5}{*}{ Weight/height (percentile) } & 5 and less & 97 & 38.2 \\
\hline & $5-10$ & 7 & 2.7 \\
\hline & $10-25$ & 75 & 29.1 \\
\hline & $25-50$ & 38 & 15 \\
\hline & 50 and more & 38 & 15 \\
\hline \multirow[t]{2}{*}{$\mathrm{FTT}$} & No & 200 & 77.2 \\
\hline & Yes & 59 & 22.8 \\
\hline \multirow[t]{4}{*}{ Wasting } & Normal & 164 & 63.3 \\
\hline & Mild & 11 & 4.2 \\
\hline & Moderate & 22 & 8.5 \\
\hline & Severe & 62 & 23.9 \\
\hline \multirow[t]{2}{*}{ Waterlow wasting } & No & 175 & 67.6 \\
\hline & Yes & 84 & 32.4 \\
\hline \multirow[t]{2}{*}{ GERD } & No & 234 & 90.3 \\
\hline & Yes & 25 & 9.7 \\
\hline \multirow[t]{2}{*}{ Occurrence of constipation } & No & 226 & 87.3 \\
\hline & Yes & 33 & 12.7 \\
\hline \multirow{2}{*}{$\begin{array}{l}\text { Usual intake is }<50 \text { of Energy } \\
\text { RDA needs pre-admission }\end{array}$} & No & 179 & 69.1 \\
\hline & Yes & 80 & 30.9 \\
\hline
\end{tabular}

$N$ number, $F T T$ failure to thrive, GERD gastroesophageal reflux disease mean (SD) of weight loss upon discharge from ICU was $6.3(4.3) \mathrm{kg}$ (range 1-26). The mean (SD) serum albumin was 39.3 (6.16) $\mathrm{mg} / \mathrm{dl}$, with (range 23-63). The total lymphocyte score mean (SD) was $3.1(2.37) \mu \mathrm{l}$, and median of 2.90 (range 1-13.0 $\mu \mathrm{l}$ ). The PNI mean (SD) was 55 (12.8), median 54 (range 30 to 115) (Figs. 1 and 2).

\section{Effect of the nutritional status on the infection rate in the ICU}

In total, $41.5 \%$ of the study sample developed an infection after the surgery. The number of patients who were $\mathrm{NPO}$ in the ICU was significantly higher in the infected group with a mean (SD) of 4.12 (5.5) days compared to the non-infected group with mean (SD) 2.17 (2.6) (95\% confidence intervals: $-3.29-0.60, P$ value $<0.05$ ) (Table 2). The following variable were found to be statistically significant by univariate analysis comparing infected and non-infected groups with $P$ values $<0.05$ : ICU LOS, hospital LOS, ventilation duration, age of patients on admission, total NPO days in ICU, total number of days to reach energy RDA, and \% RDA (Table 2). There was no significant relation between PNI and infection rate. PNI mean was $43 \pm 16.8$ and $40 \pm 15.8$ in noninfected and infected patients respectively $(P=0.14)$. There was no statistically significant relationship between infection and other parameters of malnutrition status like failure to thrive, weight, height, weight/height, maturity, and Waterlow wasting.

\section{Effect of the nutritional status on the length of ICU stay}

The mean length of stay (LOS) in ICU for all study cases was $10.5 \pm 19.6$ days, the median 5 days (range 1-203). Length of ICU stay mean was significantly higher among the severe malnutrition patients $P$ value (0.049), also the weight and height percentile have a significant effect on the ICU LOS ( $P$ value 0.009 and 0.045 ) respectively, meaning that malnutrition can significantly prolong the ICU stay. The linear regression analysis showed factors that significantly affect ICU length of stay were the age of the patient on admission, ventilation hours, the total number of NPO days, and the number of days to achieve RDA (Table 3). After testing many levels of PNI values, we noted that PNI more $\geq 55$ was able to discriminate 123 patients $(47.5 \%)$ with shorter ventilation time $(P$ $0.048)$, and shorter ICU stay $(P$ 0.036) in comparison to other cases with PNI $<55$ (Table 4).

\section{The effect of the malnutrition status of the patients' pre-admission on the other factors}

In total, $32.4 \%$ of the patients suffered from severe malnutrition pre-admission (Table 1). The patients with the nutritional intake of $<50$ RDA pre-admission 

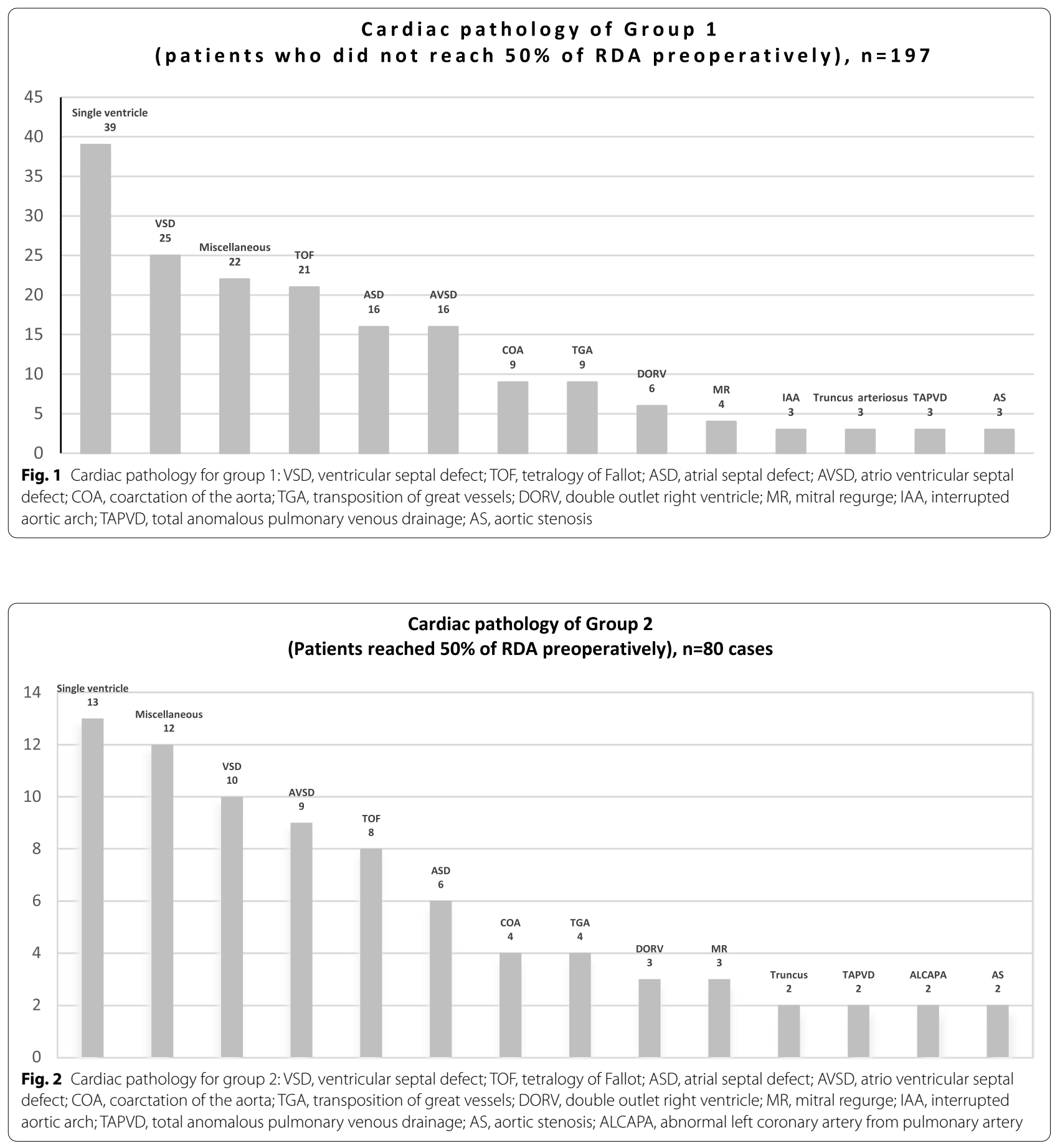

(69\%) were the cases who had more malnutrition with a $P$ value of 0.0001 . We found that patients with severe malnutrition were kept NPO longer (mean $3.40 \pm 5.56$ days) than the non-severe malnutrition patients (mean $1.82 \pm 1.45$ days), confidence intervals ( $95 \%$ CI $-0.05-$ $3.21)$ and $P$ value (0.003).

\section{Mortality}

Total postoperative mortality in the study group was 11 patients (4.2\%) (Table 1). Mortality was showing significant statistical correlation with the total NPO days $(P$ 0.03 ), and number of days to achieve recommended daily allowance RDA ( $P$ 0.05). 
Table 2 Relationship between infection and continuous risk factors

\begin{tabular}{|c|c|c|c|c|c|c|c|}
\hline & \multicolumn{4}{|c|}{ Infection } & \multirow{2}{*}{\multicolumn{2}{|c|}{$95 \mathrm{Cl}$}} & \multirow[t]{3}{*}{$P$ value } \\
\hline & \multicolumn{2}{|c|}{$\begin{array}{l}\text { No infection, } N=183 \\
\text { patients }\end{array}$} & \multicolumn{2}{|c|}{ Infection, $N=76$ patients } & & & \\
\hline & Mean & SD & Mean & SD & Lower & Upper & \\
\hline Length of ICU stay (days) & 6.79 & 7.96 & 19.51 & 32.36 & -20.20 & -5.23 & 0.001 \\
\hline Length of hospital stay (days) & 25.81 & 57.57 & 46.66 & 47.81 & -32.85 & -5.78 & 0.005 \\
\hline Ventilation hours & 50.55 & 96.25 & 172.2 & 324.76 & -199.7 & -43.58 & 0.003 \\
\hline Age of patients on admission (weeks) & 22.10 & 31.26 & 11.76 & 17.53 & 4.28 & 16.38 & 0.001 \\
\hline Total NPO days in ICU & 2.17 & 2.69 & 4.12 & 5.59 & -3.29 & -0.60 & 0.005 \\
\hline Total number of days to reach energy RDA & 4.00 & 6.73 & 6.72 & 7.04 & -4.60 & -0.83 & 0.005 \\
\hline$\%$ RDA & 82.10 & 38.07 & 93.68 & 34.06 & -21.17 & -1.98 & 0.018 \\
\hline
\end{tabular}

$N$ number, $M$ mean, $S D$ standard deviation, $C I$ confidence interval, ICU intensive care unit, NPO nil per os, RDA recommended daily allowance, \% RDA percentage of total calories based on the RDA for age and sex of each patient for all days in PCICU divided by number PCICU days and then averaged over the number of patients

Table 3 Relationship between ICU stay and continuous risk factors

\begin{tabular}{|c|c|c|c|c|c|c|c|}
\hline & \multicolumn{4}{|c|}{ ICU stay categorized } & \multirow{2}{*}{\multicolumn{2}{|c|}{$95 \mathrm{Cl}$}} & \multirow[t]{3}{*}{$P$ value } \\
\hline & \multicolumn{2}{|c|}{$\begin{array}{l}\text { Normal (less than } 3 \text { days), } \\
N=90 \text { patients }\end{array}$} & \multicolumn{2}{|c|}{$\begin{array}{l}\text { Prolonged (more than } 3 \\
\text { days), } N=165 \text { patients }\end{array}$} & & & \\
\hline & $M$ & SD & $M$ & SD & Lower & Upper & \\
\hline Length of hospital stay (days) & 1.94 & 0.90 & 15.32 & 23.16 & -25.90 & -12.69 & 0.000 \\
\hline Ventilation hours (days) & 14.85 & 24.85 & 122.35 & 237.12 & -144.65 & -70.34 & 0.000 \\
\hline Age of patients on admission (weeks) & 31.86 & 35.68 & 12.10 & 20.43 & 11.70 & 27.80 & 0.000 \\
\hline Total NPO days in ICU & 1.92 & 3.163 & 3.19 & 4.17 & -2.191 & -0.339 & 0.008 \\
\hline Days to reach energy RDA & 2.46 & 5.01 & 6.10 & 7.51 & -5.198 & -2.086 & 0.000 \\
\hline$\%$ RDA & 73 & 40.12 & 92.23 & 33.76 & -29.13 & -9.32 & 0.000 \\
\hline
\end{tabular}

$N$ number, $M$ mean, $S D$ standard deviation, $C l$ confidence interval, ICU intensive care unit, NPO nil per os, RDA recommended daily allowance, $\%$ RDA percentage of total calories based on the RDA for age and sex of each patient for all days in PCICU divided by number PCICU days and then averaged over the number of patients

Table 4 Relationship between PNI 55 and continuous risk factors

\begin{tabular}{|c|c|c|c|c|c|c|c|}
\hline & \multicolumn{4}{|l|}{ PNI 55} & \multirow{2}{*}{\multicolumn{2}{|c|}{$95 \mathrm{Cl}$}} & \multirow[t]{3}{*}{$P$ value } \\
\hline & \multicolumn{2}{|c|}{$<55, N=136$ patients } & \multicolumn{2}{|c|}{$\geq 55, N=123$ patients } & & & \\
\hline & M & SD & $\mathbf{M}$ & SD & Lower & Upper & \\
\hline Length of ICU stay (days) & 12.49 & 24.12 & 7.70 & 9.50 & 0.325 & 9.24 & 0.036 \\
\hline Length of hospital stay (days) & 35.96 & 70.00 & 24.92 & 27.20 & -1.82 & 23.91 & 0.092 \\
\hline Ventilation hours & 104.52 & 247.02 & 57.99 & 96.46 & 0.474 & 92.58 & 0.048 \\
\hline Age of patients on admission (weeks) & 20.06 & 32.68 & 17.14 & 21.68 & -3.88 & 9.72 & 0.399 \\
\hline Total NPO days in ICU & 3.14 & 4.25 & 2.33 & 3.46 & -0.15 & 1.78 & 0.099 \\
\hline Days to reach energy RDA & 4.96 & 7.59 & 4.45 & 5.70 & -1.14 & 2.16 & 0.544 \\
\hline$\%$ RDA & 86.53 & 39.53 & 84.61 & 34.74 & -7.40 & 11.25 & 0.685 \\
\hline
\end{tabular}

$P N I$ prognostic nutritional index, $N$ number, $M$ mean, $S D$ standard deviation, $C l$ confidence interval, intensive care unit, $N P O$ nil per os, $R D A$ recommended daily allowance, \% RDA percentage of total calories based on the RDA for age and sex of each patient for all days in PCICU divided by number PCICU days and then averaged over the number of patients

\section{Discussion}

Malnutrition is the main reason for infants and children mortality throughout the world [21,22], and it is directly responsible for approximately 300,000 childhood deaths per year [23]. Nutritional deficiency also increases the risk of mortality in an intensive care population. Proteinenergy malnutrition significantly affects the human physiology and these effects include cardiac, renal, hepatic 
function, humoral, and cellular immunity [10]. Gastrointestinal intolerance is frequently observed in critically ill children who receive insufficient calories and proteins. In addition to that, there is a restriction to fluid intake, and interruptions in enteral nutrition in order to administer medications resulting in a further decrease in protein and calories intake [24]. In our sample population, we recognized 9.7\% incidence of GERD and constipation in $12.7 \%$.

Vaidyanathan et al. reported a prospective observational cohort that included 476 patients with CHD less than 16 months old who underwent cardiac surgery. Fifty-nine percent (weight), 26.3\% (height), and 55.9\% (weight/height) of patients had Z-scores of $\leq-2$ at presentation, Z-scores of $\leq-3$ for weight, height, and weight/height were observed in $27.7 \%, 10.1 \%$, and $24.2 \%$ patients, respectively. Three months follow-up post cardiac surgery showed statistically significant improvement in all parameters of weight $P<0.001$, height $P 0.04$, weight/height and in-hospital mortality of $2.7 \%$ [25]. Ratanachu-ek et al. conducted a prospective cohort study in 161 pediatric patients with CHD who underwent cardiac surgery. The nutritional status of the patients before surgery was defined as normal 57\%, malnutrition $40 \%$, and over-nutrition 3\%. Malnutrition included underweight $28 \%$, wasting $22 \%$, and stunting $16 \%$. Post-cardiac surgery, the means of Z-score of weight for age (WFA), weight for height (WFH), and height for age (HFA) were significantly increased and the prevalence of underweight and wasting were decreased to $17 \%$ and $6 \%$ respectively, which was statistically significant compared to the baseline $(p<0.05)$ [4]. In our current study, we have $32.4 \%$ of the patients suffered from severe malnutrition preadmission; the abnormality of weight and height of less than $5 \%$ of the sample were $48.2 \%$ and $36.3 \%$ respectively. Lower weight-for-age and weight-for-height have been reported to be associated with poor outcomes in pediatric critical care; these results correlate with our findings of increased risk of infection in patients who were kept NPO for long time, ventilation time, increased ICU LOS, and even risk of mortality. Okoromah and colleagues reported a $41 \%$ prevalence of "wasting" defined by WHO as weight-for-height $Z$ score $<-2$. Among pediatric cardiac patients with CHD in Lagos, Nigeria, malnutrition has been linked to increased morbidity and mortality, as indicated by frequent hospitalization, poor surgical outcomes, persistent impairment of somatic growth, and increased mortality [26]. Monique Radman et al. concluded in their study that there is a strong correlation between acute and chronic malnourishment and worse clinical outcomes post-cardiac repair in children undergoing surgery for CHD, specifically the ICU length of stay and the ventilation time, these findings strongly correlate with our findings in the current study [27]. PNI is one of the reliable tools to assess the nutritional status of pediatric patients in the perioperative period. In our present study, PNI was significantly correlating with the ventilation time, and the PICU LOS; these results are correlating with Wakita and his colleagues who concluded in their study that preoperative PNI score is a predictor of the length of stay in the PICU for pediatric patients after cardiac surgery; patients with low PNI score had PICU stay 5 days more than patients with high PNI score. A cut-off point of 55 for the PNI score proved to be a reliable predictor which is the same cut-off point we used and confirmed the strong correlation with ICU length of stay and ventilation time [13]. Buzby et al. proved in their PNI using albumin level, triceps skinfold thickness, serum transferrin level, and cutaneous delayed hypersensitivity reactivity predicts incidence of postoperative infectious complications, septicemia, and mortality, but this study was in adults [28], in our current study using the Onodera's PNI we could not prove any correlation of PNI with the risk of infection. The relation between optimal delivery of enteral nutrition and clinical outcomes, including mortality, has been well described in multiple studies [29, 30], Manoj et al. demonstrated in their study the feasibility of early enteral nutrition for patients with congenital cardiac malformations, postoperatively with mother's milk. Providing fortification in the form of a calorie dense expressed breast milk to these infants is tolerated and also benefits in better postoperative recovery with less incidence of infection and shorter ICU stay [31]. In the current study, it was very obvious that patients who were kept NPO longer suffered more severe malnutrition, and there was a strong correlation between the NPO duration and the inadequate intake expressed as RDA less than $50 \%$ with the incidence of infection, ventilation duration, and postoperative mortality. Our study has many limitations that include single center experience. Also, late referral of some of our patients could have affected negatively their nutritional status and the results of our study. Furthermore, due to many confounding factors that include type of surgery, complexity of surgery, bypass time, presence of residual lesions, feeding tolerance, and difficulty to adapt a uniform pattern in initiating feeding in postoperative cardiac children that may affect the study result and conclusion. Though we attempt to introduce enteral feeding in majority of our cases as early as possible, on many occasions the feeding had to be interrupted or replaced with parenteral feeding in children with complex cardiac surgery or in neonates and small infants who frequently suffer from feeding intolerance or other complication such as necrotizing enterocolitis or chylothorax that can affect their ability to absorb or to be fed through normal gastrointestinal tract. We believe that multi-center with nutritional protocol-based study 
is recommended to minimize bias and limitation of small number of cases in single center study. Also, such multicenter study will verify the discriminatory PNI 55 finding that we proposed in our study based on analysis of our group of patients.

\section{Conclusion}

There is a high prevalence of severe malnutrition in patients with congenital heart disease preoperatively, and they have a further loss in weight postoperatively. Malnutrition increase ventilation time, pediatric intensive care length of stay, and consequently the cost. PNI may be a useful index for nutritional assessment and potential prediction for PICU length of stay. Delayed postop enteral feeding may increase the risk of infection and mortality. Adequate preoperative assessment of the nutritional status for pediatric cases with congenital heart diseases, and putting plans to improve it, may help to improve the postoperative outcome.

\begin{abstract}
Abbreviations
ICU: Intensive care unit; CHD: Congenital heart disease; PCICU: Pediatric cardiac intensive care unit; PNI: Prognostic nutritional index; NPO: Nil per os; RDA: Recommended daily allowance; LOS: Hospital length of stay; WFA: Weight for age; WFH: Weight for height; HFA: Height for age; GERD: Gastro esophageal reflux disease; WHO: World Health Organization; M: Mean; N: Number; SD: Standard deviation; Cl: Confidence interval; FTT: Failure to thrive; RACHs: Risk adjustment for congenital heart surgery.
\end{abstract}

\section{Acknowledgements}

Not applicable

\section{Authors' contributions}

SI: Corresponding author, contributed in the study design, data collection, writing the manuscript, and submission for publication. AM: Contributed in the study design, and data collection. NR: Data collection, calculating PNI for patients in the nutrition clinic and PCICU. RA: Study design, manuscript review. MK: Study design, manuscript review. All authors have read and approved the manuscript.

\section{Funding}

There was no funding for this study.

\section{Availability of data and materials}

The datasets used and/or analyzed during the current study are available from the corresponding author on reasonable request.

\section{Declarations}

\section{Ethics approval and consent to participate}

This study was approved on 15 August 2013 (reference IRBC/212/13) by the Institutional Review Board (IRB) of King Abdullah International Medical Research Center. Appropriate written consent was obtained before including any patient in the study.

\section{Consent for publication}

Not applicable.

\section{Competing interests}

The authors declare that they have no competing interests.
Received: 23 December 2020 Accepted: 10 October 2021

Published online: 29 November 2021

\section{References}

1. Save the Children (2011) Hunger. Save the Children, London Available online: http://www.savethechildren.org.uk/en/hunger.htm (Accessed on 16 Nov 2011)

2. Mitchell IM, Logan RW, Pollock JC et al (1995) Nutritional status of children with congenital heart disease. Br Heart J 73(3):277-283

3. Vieira TC, Trigo M, Alonso RR et al (2007) Assessment of food intake in infants between 0 and 24 months with congenital heart disease. Arq Bras Cardiol 89(4):219-224

4. Ratanachu-Ek S, Pongdara A (2011) Nutritional status of pediatric patients with congenital heart disease: pre- and post cardiac surgery. J Med Assoc Thail 94(Suppl 3):S133-S137

5. Pillo-Blocka F, Adatia I, SharieffW et al (2004) Rapid advancement to more concentrated formula in infants after surgery for congenital heart disease reduces duration of hospital stay: a randomized clinical trial. J Pediatr 145(6):761-766

6. Kyle UG, Genton L, Pichard C (2005) Hospital length of stay and nutritional status. Curr Opin Clin Nutr Metab Care 8(4):397-402

7. Mota EM, Garcia PC, Piva JP et al (2002) The influence of poor nutrition on the necessity of mechanical ventilation among children admitted to the pediatric intensive care unit. J Pediatr 78(2):146-152

8. Huse DM, Feldt RH, Nelson RA et al (1975) Infants with congenital heart disease. Food intake, body weight, and energy metabolism. Am J Dis Child 129(1):65-69

9. Salzer HR, Haschke F, Wimmer M et al (1989) Growth and nutritional intake of infants with congenital heart disease. Pediatr Cardiol 10(1):17-23

10. Eskedal LT, Hagemo PS, Seem E et al (2008) Impaired weight gain predicts risk of late death after surgery for congenital heart defects. Arch Dis Child 93(6):495-501

11. Braudis NJ, Curley MA, Beaupre K et al (2009) Enteral feeding algorithm for infants with hypoplastic left heart syndrome poststage I palliation. Pediatr Crit Care Med 10(4):460-466

12. Jadcherla SR, Vijayapal AS, Leuthner S (2009) Feeding abilities in neonates with congenital heart disease: a retrospective study. J Perinatol 29(2):112-118

13. Wakita M, Fukatsu A, Amagai T (2011) Nutrition assessment as a predictor of clinical outcomes for infants with cardiac surgery: using the prognostic nutritional index. Nutr Clin Pract 26(2):192-198

14. Nozoe T, Ninomiya M, Maeda T et al (2010) Prognostic nutritional index: a tool to predict the biological aggressiveness of gastric carcinoma. Surg Today 40(5):440-443

15. Anderson JB, Beekman RH 3rd, Border WL et al (2009) Lower weight-forage $z$ score adversely affects hospital length of stay after the bidirectional Glenn procedure in 100 infants with a single ventricle. J Thorac Cardiovasc Surg 138(2):397-404 e391

16. Irving SY, Simone SD, Hicks FW et al (2000) Nutrition for the critically il child: enteral and parenteral support. AACN Clin Issues 11(4):541-558 quiz 637-548

17. WHO Multicentre Growth Reference Study Group (2006) WHO child growth standards: length/height-for-age, weight-for-age, weight-forlength, weight-for-height and body mass index-for-age: methods and development. World Health Organization, Geneva (312 pages)

18. Waterlow JC (1972) Classification and definition of protein-calorie malnutrition. Br Med J 3(5826):566-569

19. Waterlow JC (1973) Note on the assessment and classification of proteinenergy malnutrition in children. Lancet 2(7820):87-89

20. Onodera T, Goseki N, Kosaki G (1984) Prognostic nutritional index in gastrointestinal surgery of malnourished cancer patients. Nihon Geka Gakkai Zasshi 85(9):1001-1005

21. Pelletier DL (1994) The relationship between child anthropometry and mortality in developing countries: implications for policy, programs and future research. J Nutr 124(10 Suppl):2047S-2081S

22. Kielmann AA, McCord C (1978) Weight-for-age as an index of risk of death in children. Lancet 1(8076):1247-1250 
23. Muller O, Krawinkel M (2005) Malnutrition and health in developing countries. CMAJ 173(3):279-286

24. Mehta NM, Duggan CP (2009) Nutritional deficiencies during critical illness. Pediatr Clin N Am 56(5):1143-1160

25. Vaidyanathan B, Nair SB, Sundaram KR et al (2008) Malnutrition in children with congenital heart disease (CHD) determinants and short term impact of corrective intervention. Indian Pediatr 45(7):541-546

26. Okoromah CA, Ekure EN, Lesi FE et al (2011) Prevalence, profile and predictors of malnutrition in children with congenital heart defects: a case-control observational study. Arch Dis Child 96(4):354-360

27. Radman M, Mack R, Barnoya J et al (2014) The effect of preoperative nutritional status on postoperative outcomes in children undergoing surgery for congenital heart defects in San Francisco (UCSF) and Guatemala City (UNICAR). J Thorac Cardiovasc Surg 147(1):442-450

28. Buzby GP, Mullen JL, Matthews DC et al (1980) Prognostic nutritional index in gastrointestinal surgery. Am J Surg 139(1):160-167
29. Mehta NM, Bechard LJ, Cahill N et al (2012) Nutritional practices and their relationship to clinical outcomes in critically ill children--an international multicenter cohort study*. Crit Care Med 40(7):2204-2211

30. Mikhailov TA, Kuhn EM, Manzi J et al (2014) Early enteral nutrition is associated with lower mortality in critically ill children. JPEN J Parenter Enteral Nutr 38(4):459-466

31. Sahu MK, Singal A, Menon R et al (2016) Early enteral nutrition therapy in congenital cardiac repair postoperatively: a randomized, controlled pilot study. Ann Card Anaesth 19(4):653-661

\section{Publisher's Note}

Springer Nature remains neutral with regard to jurisdictional claims in published maps and institutional affiliations.

\section{Submit your manuscript to a SpringerOpen ${ }^{\circ}$ journal and benefit from:}

- Convenient online submission

- Rigorous peer review

- Open access: articles freely available online

- High visibility within the field

- Retaining the copyright to your article

Submit your next manuscript at springeropen.com 